\title{
Impact of Social Determinants on the Burden of Asthma and Eczema: Results from a US Patient Survey
}

\author{
Don A. Bukstein · Adam Friedman · Erika Gonzalez Reyes · \\ Mary Hart · Bridgette L. Jones · Tonya Winders
}

Received: July 30, 2021 / Accepted: December 14, 2021 / Published online: January 24, 2022

(C) The Author(s) 2022

\section{ABSTRACT}

Introduction: Little is known about how patients with asthma and eczema perceive their medical care and burden of disease. A survey was conducted to evaluate the perceptions among the general patient population with

Don A. Bukstein, Adam Friedman, Erika Gonzalez Reyes, Mary Hart, and Bridgette L. Jones contributed equally to this work and are listed alphabetically.

Supplementary Information The online version contains supplementary material available at https:// doi.org/10.1007/s12325-021-02021-0.

D. A. Bukstein

Allergy, Asthma \& Sinus Center, Milwaukee, WI, USA

A. Friedman

Department of Dermatology, George Washington

School of Medicine and Health Sciences,

Washington, DC, USA

E. Gonzalez Reyes

Department of Internal Medicine, University of

Texas Health Science Center, San Antonio, TX, USA

M. Hart · T. Winders ( $\square)$

Allergy \& Asthma Network, Vienna, VA, USA

e-mail: twinders@AllergyAsthmaNetwork.org

B. L. Jones

Children's Mercy Kansas City \& University of

Missouri-Kansas City School of Medicine, Kansas

City, MO, USA asthma and/or eczema regarding disease and treatment burden and barriers to adequate care. Methods: An online survey was completed by market research panelists in the USA between March 24, 2020 and April 6, 2020. Eligible participants were at least 18 years of age and endorsed a diagnosis of asthma and/or eczema. Survey responses are described for all participants, by designated racial/ethnic groups, and by income level.

Results: In all, 841 participants completed the survey (asthma, $n=554$; eczema, $n=398$; both, $n=111$; White, $n=421$; Black, $n=252$; Hispanic, $n=95$; low income [less than $\$ 15,000$ / year], $n=99$; higher income [at least $\$ 15,000$ / year], $n=713$ ). More Black and Hispanic participants than White participants, and more participants with low income than higher income, endorsed health literacy as a barrier (e.g., filling out official documents, understanding written materials). Participants with low income were less likely than participants with higher income to have an asthma action plan ( $42 \%$ vs $53 \%$, respectively) and to discuss asthma control with their healthcare provider (54\% vs 69\%). Black and Hispanic participants were more likely than White participants to have an emergency department visit (52\% and $49 \%$ vs $31 \%$, respectively) or hospitalization (31\% and $39 \%$ vs $16 \%$ ) for asthma within the last 12 months. Participants reporting low income indicated that they experienced eczema symptoms more frequently than participants 
with higher income; 35\% of low-income participants vs $15 \%$ of higher-income participants reported that they had not tried any eczema treatments. Participants in all racial/ethnic and income-level groups reported that their asthma or eczema impacted their lifestyle and daily activities.

Conclusion: More effective and culturally informed communication and education strategies to improve health information uptake and shared decision-making are needed to reduce the burdens of disease and treatment in highly impacted populations.

\section{PLAIN LANGUAGE SUMMARY}

Disparities in asthma and eczema outcomes have been described in various populations. However, little is known about how these patient populations perceive their disease management or disease burden. A survey of 841 adults across diverse demographic groups in the USA with asthma and/or eczema was conducted to evaluate overall perceived disease burden and to specifically understand burden experiences by marginalized populations. In general, all participants indicated that asthma and eczema have a negative physical, emotional, and social impact on their lives. Some participants who identified as Black or Hispanic, and those with low income (less than $\$ 15,000 /$ year), indicated greater difficulties in filling out paperwork or understanding written materials related to their condition than White participants or those with higher incomes. Black and Hispanic participants tended to receive asthma care in the emergency department or urgent care more than White participants and had more emergency department visits and hospitalizations than White Participants. Participants with low income were less likely to discuss their asthma management with their doctor than those with higher incomes and also indicated potential undertreatment of eczema. These results indicate that Black, Hispanic, or low-income patients may experience barriers to health equity. These barriers include lack of effective communication methods and materials to meet the needs of all patients, as well as the overall lack of quality healthcare access. These challenges must be addressed to overcome social disparities in health.

Keywords: Asthma; Atopic dermatitis; Burden; Disparities; Eczema; Health equity

\section{Key Summary Points}

Why carry out this study?

Understanding the perceptions among the general patient population with asthma and/or eczema regarding disease and treatment burden and barriers to adequate care will help healthcare programs and policy makers to develop targeted efforts to improve outcomes.

\section{What was learned from the study?}

The burden of asthma and eczema disease and treatment impacts patients differently and is associated with social determinates of health.

Some Black and Hispanic patients endorse health literacy barriers and experience more emergency department visits and hospitalizations than White patients.

Some patients with low income endorse health literacy barriers, have fewer asthma management discussions with their healthcare provider, and have undermanaged eczema than patients with higher incomes.

Interventions and materials to support health literacy and shared decisionmaking conversations are needed to mitigate these observed disparities and overall move towards opportunity for health equity in Black, Hispanic, and lowincome patients with asthma and eczema. 


\section{INTRODUCTION}

Asthma and atopic dermatitis (eczema) are closely associated and often concomitant conditions. Disparities in outcomes for these conditions have been described in various populations. For example, individuals who identify as Black or Hispanic have higher rates of emergency department (ED) visits and hospitalizations for asthma and eczema compared with non-Hispanic White individuals [1-4]. Likewise, those who report low income also have higher rates of ED visits and hospitalizations compared with those with higher incomes $[2,4]$. Severe eczema is associated with a lack of insurance, denial of eczema prescriptions by insurers, and the costs of treatments for eczema [4]. Furthermore, eczema may not be readily identified by healthcare providers (HCP) who may lack experience and training in diagnosis of skin conditions in people of color [5]. This gap in medical knowledge may lead to increased morbidity due to missed and/or delayed diagnosis and treatment [6]. Individuals from racially/ethnically marginalized populations and those who report lower income also have reported lower rates of health insurance enrollment than those from non-marginalized groups, which impacts overall access to quality healthcare $[1,2]$. For patients who are underinsured or uninsured, the ED often becomes the fallback for medical care [1]. These social determinants of health are known contributors to observed asthma and allergic disease disparities $[7,8]$.

The physical, emotional, and healthcare burden of asthma and eczema is well documented. Patients report that these conditions can impact daily activities, quality of life, sleep quality, and work/school productivity [9-12]. Both asthma and eczema are chronic conditions which often require lifelong treatment to achieve and maintain control, creating additional costs and emotional burden [11, 13-15]. In addition, asthma has significant financial impact because of the need for frequent office visits for continued monitoring of acute care, as well as ED visits, and hospitalizations associated with exacerbations [16, 17]. Healthcare utilization is also higher among patients with eczema compared with the general population $[9,13]$.

While the overall burden of asthma and eczema is well known, there is little information about how patients perceive their disease, medical care, and burden of disease. A survey was conducted to evaluate the perceptions among the general patient population with asthma and/or eczema regarding disease and treatment burden and barriers to adequate care.

\section{METHODS}

\section{Survey Development}

The survey was developed to assess how patients with asthma and/or eczema perceive their disease and treatment burden in terms of physical, emotional and social health, how they perceive their asthma- and eczema-related medical care, and to identify barriers to care.

\section{Participant and Survey Details}

The survey was posted online for access through panels consisting of individuals in the general population of the USA between March 24, 2020 and April 6, 2020. Panels were hosted by Dynata, a global online market research firm based in the USA. Panelists accessed the survey through the Dynata dashboard or platform for their respective panel. Participants were required to be at least 18 years of age and indicate that they were diagnosed by a medical professional with either asthma or eczema.

The 94-question online survey shown in Supplemental Table E1 was self-completed by each participant. For completing the survey, participants were given an incentive that varied by panel. Typically, the incentives were "panel points" that could be exchanged for goods or services through the panelists' respective panel dashboard.

The survey was reviewed by an institutional review board and was granted exemption status. All participants provided written informed 
consent at the time of survey participation for the use of their survey responses.

\section{Analysis}

In addition to responses in the overall participant population, survey responses were analyzed by self-reported race and ethnicity (White, Black, or Hispanic) and self-reported income status (low income, income less than $\$ 15,000$ / year; higher income, income at least $\$ 15,000$ / year). Participants who identified as Hispanic were not further divided into Hispanic racial categories (i.e., Black Hispanic) for the analysis. Statistical significance between Black or Hispanic participants versus White participants, and for participants with low income versus participants with higher income, was defined as non-overlapping 95\% confidence intervals.

\section{RESULTS}

\section{Demographic and Disease Characteristics}

A total of 841 participants completed the survey; 554 (66\%) participants reported an asthma diagnosis and 398 (47\%) reported an eczema diagnosis (111 [13\%] participants reported both diagnoses). The majority (76\%) of participants were women, $41 \%$ were aged 55 or over, $50 \%$ identified as White, 30\% identified as Black, and $11 \%$ identified as Hispanic (Table 1). Asthma was reported by a higher percentage of participants identifying as Black or Hispanic than White participants (85\% and $87 \%$ vs $48 \%$, respectively), whereas eczema was reported by a lower percentage of participants identifying as Black or Hispanic than White participants (21\% and $19 \%$ vs $71 \%$, respectively; Table 1 ).

\section{Socioeconomic Characteristics}

Among all participants, 99 (12\%) reported income less than $\$ 15,000 /$ year (low income). Of the participants with low income, a higher percentage were female and a higher percentage identified as Black compared with participants with higher income (Table 1). Asthma was reported by a higher percentage of participants with low income and eczema was reported by a lower percentage in this group compared with participants with higher income (Table 1).

\section{Healthcare Access and Communication}

Black, Hispanic, and White participants reported similar frequencies for having employerprovided health insurance $(31 \%, 38 \%$, and $33 \%$, respectively). Black participants were more likely to have Medicaid or other state-provided health insurance than White participants (37\% vs $15 \%$, respectively). Only $6 \%$ of participants with low income reported having employerprovided health insurance, compared with 36\% of participants with higher income. Participants with low income were more likely to have Medicaid or other state-provided health insurance than participants with higher income ( $72 \%$ vs $17 \%$, respectively). In all, $6 \%$ of all participants did not have any health insurance. The primary reported reason for lack of health insurance was the inability to afford it.

A similar percentage of Black, Hispanic, and White participants $(43 \%, 48 \%$, and $43 \%$ respectively) reported non-adherence to medication prescribed by their HCP. A higher percentage of participants with low income reported non-adherence to medication than participants with higher income (52\% vs $40 \%$, respectively). Among those who reported nonadherence, reasons for not taking a prescribed medication were the medication was too expensive (34\%), the medication was not covered by insurance $(23 \%)$, the prescription took too long to fill $(12 \%)$, or other reasons such as side effects, feeling better, forgetting, etc. (31\%).

A higher percentage of Black participants (28\%) and Hispanic participants (33\%) than White participants (14\%), and a higher percentage of participants with low income (42\%) than higher income (17\%), reported that they lacked transportation in the previous 12 months to get to appointments, work, or get things they need. More Black and Hispanic participants than White participants, and more participants with low income than higher income, reported that they were at least 
Table 1 Demographics of survey participants

\begin{tabular}{|c|c|c|c|c|c|c|c|c|}
\hline Demographic, \% & $\begin{array}{l}\text { Total } \\
\text { participants } \\
(N=841)\end{array}$ & $\begin{array}{l}\text { Total with } \\
\text { asthma } \\
(n=554)\end{array}$ & $\begin{array}{l}\text { Total with } \\
\text { eczema } \\
(n=398)\end{array}$ & $\begin{array}{l}\text { White } \\
(n=421)\end{array}$ & $\begin{array}{l}\text { Black } \\
(n=252)\end{array}$ & $\begin{array}{l}\text { Hispanic } \\
(n=95)\end{array}$ & $\begin{array}{l}\text { Low } \\
\text { income } \\
(n=99)\end{array}$ & \\
\hline Female & 76 & 79 & 71 & 72 & $80^{\mathrm{a}}$ & $84^{\mathrm{a}}$ & 88 & 73 \\
\hline \multicolumn{9}{|l|}{ Age, years } \\
\hline $18-24$ & 13 & 17 & 6 & 6 & $27^{\mathrm{a}}$ & $23^{a}$ & 18 & 12 \\
\hline $25-34$ & 16 & 17 & 15 & 13 & 18 & $22^{\mathrm{a}}$ & 12 & 16 \\
\hline $35-44$ & 16 & 16 & 15 & 12 & 15 & $22^{a}$ & 17 & 16 \\
\hline $45-54$ & 16 & 16 & 15 & 17 & $10^{\mathrm{a}}$ & 15 & 22 & 14 \\
\hline $55-64$ & 20 & 19 & 22 & 22 & 19 & 14 & 23 & 19 \\
\hline $65+$ & 21 & 16 & 28 & 30 & $12^{\mathrm{a}}$ & $4^{\mathrm{a}}$ & $7^{\mathrm{b}}$ & \\
\hline
\end{tabular}

Ethnicity

\begin{tabular}{|c|c|c|c|c|c|c|c|c|}
\hline White & 50 & 36 & 75 & 100 & - & 23 & 43 & 51 \\
\hline Black & 30 & 38 & 14 & - & 100 & 25 & $42^{b}$ & 29 \\
\hline Native & 3 & 4 & 2 & - & - & 7 & 1 & 4 \\
\hline \multicolumn{9}{|l|}{ American } \\
\hline Asian & 8 & 10 & 5 & - & - & 3 & 4 & 9 \\
\hline Native & 1 & 1 & 1 & - & - & 4 & 0 & 1 \\
\hline \multirow{2}{*}{\multicolumn{9}{|c|}{$\begin{array}{l}\text { Hawaiian/ } \\
\text { Pacific Islander }\end{array}$}} \\
\hline & & & & & & & & \\
\hline Other & 7 & 9 & 3 & - & - & 36 & 8 & 7 \\
\hline $\begin{array}{l}\text { Prefer not to } \\
\text { answer }\end{array}$ & 1 & 1 & 1 & 0 & 1 & 1 & 1 & 1 \\
\hline Hispanic & 11 & 15 & 5 & 5 & 10 & 100 & 10 & 12 \\
\hline \multicolumn{9}{|c|}{ Diagnosed conditions } \\
\hline Allergies & 62 & 69 & 58 & 63 & 59 & 65 & 68 & 61 \\
\hline Asthma & 66 & 100 & 28 & 48 & $85^{\mathrm{a}}$ & $87^{\mathrm{a}}$ & $75^{b}$ & 64 \\
\hline Eczema & 47 & 20 & 100 & 71 & $21^{\mathrm{a}}$ & $19^{\mathrm{a}}$ & 37 & 49 \\
\hline Depression & 28 & 28 & 32 & 33 & $21^{\mathrm{a}}$ & 36 & $46^{b}$ & 26 \\
\hline Hypertension & 33 & 31 & 37 & 37 & 33 & $23^{\mathrm{a}}$ & 31 & 33 \\
\hline Nasal allergies & 28 & 29 & 31 & 32 & $19^{\mathrm{a}}$ & 34 & 26 & 27 \\
\hline Psoriasis & 6 & 4 & 9 & 9 & $2^{a}$ & 4 & 5 & 6 \\
\hline Sleep apnea & 13 & 13 & 16 & 16 & $10^{\mathrm{a}}$ & 16 & 16 & 13 \\
\hline Education & & & & & & & & \\
\hline
\end{tabular}


Table 1 continued

\begin{tabular}{|c|c|c|c|c|c|c|c|c|}
\hline Demographic, \% & $\begin{array}{l}\text { Total } \\
\text { participants } \\
(\boldsymbol{N}=\mathbf{8 4 1})\end{array}$ & $\begin{array}{l}\text { Total with } \\
\text { asthma } \\
(n=554)\end{array}$ & $\begin{array}{l}\text { Total with } \\
\text { eczema } \\
(n=398)\end{array}$ & $\begin{array}{l}\text { White } \\
(n=421)\end{array}$ & $\begin{array}{l}\text { Black } \\
(n=252)\end{array}$ & $\begin{array}{l}\text { Hispanic } \\
(n=95)\end{array}$ & $\begin{array}{l}\text { Low } \\
\text { income } \\
(n=99)\end{array}$ & $\begin{array}{l}\text { High } \\
\text { income } \\
(n=713)\end{array}$ \\
\hline $\begin{array}{l}\text { Less than high } \\
\text { school }\end{array}$ & 2 & 3 & 2 & 1 & $6^{\mathrm{a}}$ & 2 & $9^{\mathrm{b}}$ & 1 \\
\hline $\begin{array}{l}\text { High school/ } \\
\text { GED }\end{array}$ & 16 & 16 & 15 & 16 & 21 & 17 & $30^{\mathrm{b}}$ & 15 \\
\hline $\begin{array}{l}\text { Some college/ } \\
\text { Associate's } \\
\text { degree }\end{array}$ & 39 & 39 & 37 & 39 & 43 & 46 & 39 & 40 \\
\hline $\begin{array}{l}\text { Bachelor's } \\
\text { degree or } \\
\text { higher }\end{array}$ & 42 & 42 & 46 & 44 & $30^{\mathrm{a}}$ & 35 & $22^{\mathrm{b}}$ & 44 \\
\hline \multicolumn{9}{|l|}{ Geographic region } \\
\hline Northeast & 18 & 17 & 20 & 21 & $14^{\mathrm{a}}$ & 19 & 13 & 18 \\
\hline Midwest & 21 & 19 & 24 & 24 & 21 & $8^{\mathrm{a}}$ & 28 & 20 \\
\hline South & 39 & 38 & 37 & 35 & $51^{a}$ & 42 & 35 & 40 \\
\hline West & 22 & 26 & 19 & 20 & $14^{\mathrm{a}}$ & $31^{\mathrm{a}}$ & 24 & 22 \\
\hline \multicolumn{9}{|l|}{ Household income } \\
\hline$<\$ 15,000$ & 12 & 13 & 9 & 10 & $17^{\mathrm{a}}$ & 11 & 100 & - \\
\hline$\$ 15,000-24,999$ & 12 & 12 & 11 & 10 & 15 & 11 & - & 14 \\
\hline$\$ 25,000-35,999$ & 12 & 12 & 14 & 12 & 14 & 11 & - & 15 \\
\hline$\$ 35,000-49,999$ & 16 & 15 & 16 & 16 & 16 & 22 & - & 18 \\
\hline$\$ 50,000-74,999$ & 22 & 22 & 22 & 21 & 18 & 25 & - & 26 \\
\hline$\$ 75,000-99,999$ & 17 & 14 & 20 & 19 & 14 & 13 & - & 20 \\
\hline$\$ 100,000+$ & 5 & 7 & 5 & 8 & 4 & 4 & - & 7 \\
\hline $\begin{array}{l}\text { Prefer not to } \\
\text { answer }\end{array}$ & 3 & 5 & 3 & 4 & 2 & 3 & - & 0 \\
\hline
\end{tabular}

${ }^{a}$ Significantly different than White participants

bignificantly different than participants with higher income

somewhat likely to need help filling out official documents (Fig. 1a). In addition, a higher percentage of Black and Hispanic participants than White participants indicated that they sometimes have problems learning about their medical conditions because of difficulty understanding written materials (Fig. 1b). Participants with low income were less likely than 
participants with higher income to indicate that they provide their HCP with all the necessary information for their care (Fig. 2a). The majority of all participants indicated that they often make sure the HCP explains anything they do not understand (Fig. 2b). However, Hispanic participants indicated that they were less likely than White participants to ask the HCP questions (Fig. 2c). A higher percentage of Black participants (40\%) and Hispanic participants $(42 \%)$ than White participants (32\%) indicated that they would definitely question the HCP's advice based on their own research.

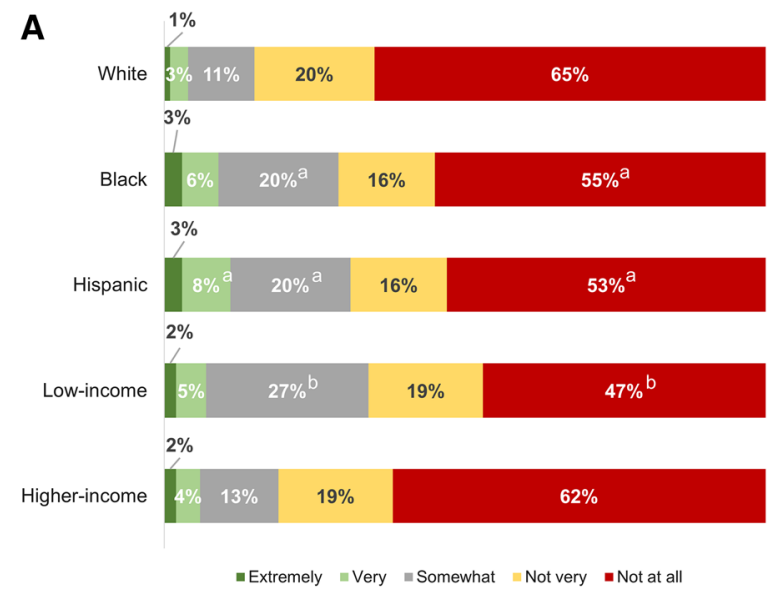

B

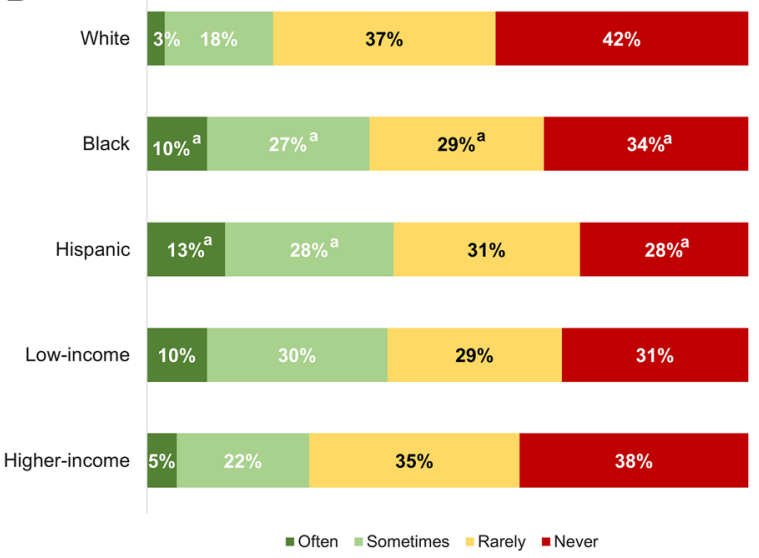

Fig. 1 Percentage of participants a likely to need help filling out official documents and $\mathbf{b}$ who have problems learning about their medical condition because of difficulty

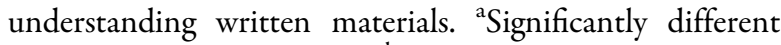
than White participants. 'bignificantly different than participants with higher income

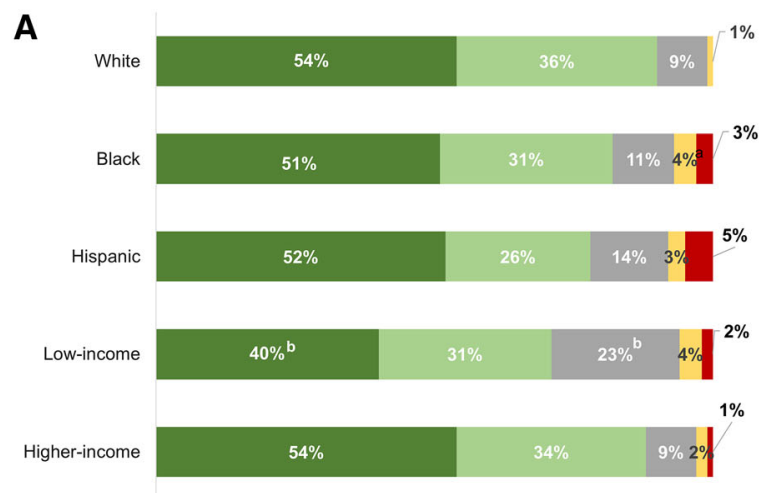

-Extremely $=$ Very $=$ Somewhat $=$ Not very $\quad$ - Not at all

B

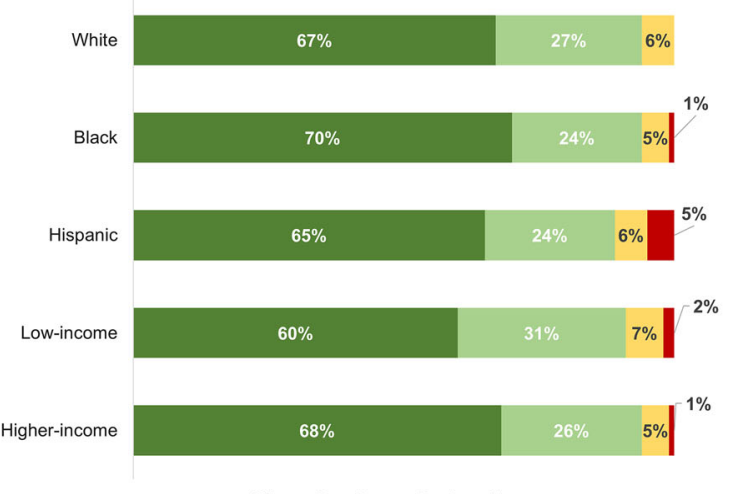

C
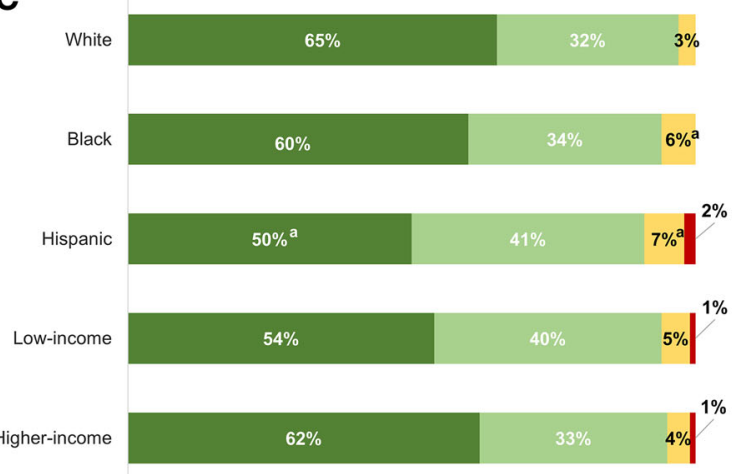

m Often $\backsim$ Sometimes $=$ Rarely $\backsim$ Never

Fig. 2 Percentage of participants a likely to give healthcare provider all information needed for care, $\mathbf{b}$ who make sure the healthcare provider explains anything they do not understand, and $\mathbf{c}$ who ask their healthcare provider questions. ${ }^{a}$ Significantly different than White participants. ${ }^{\mathrm{b}}$ Significantly different than participants with higher income 
Social, Lifestyle, and Activity Impact of Asthma and Eczema

Overall, participants indicated that their asthma and eczema can have a negative social impact (Table 2). Participants with asthma were more likely than participants with eczema to feel that they were left out of things or treated unkindly because of their condition. In contrast, participants with eczema were more likely than participants with asthma to feel embarrassed because of their condition.

Close to half of participants (43\%) reported that their asthma kept them from getting much done at work/school/home at least some of the time in the past 4 weeks. Approximately half (53\%) of participants also reported that they had to change their lifestyle and 15\% changed jobs because of their asthma. Participants commonly had to modify or stop doing activities because of asthma, particularly exercising or sports (Table 3 ).

Approximately one-third of participants with eczema (32\%) reported that their skin had been itchy, sore, painful or stinging either "very much" or "a lot" in the last week (Table 4). A higher percentage of Hispanic participants than White participants reported that eczema had negatively impacted several aspects of their life "very much" or "a lot" in the past week (Table 4).

\section{Patient Perception of Asthma}

In general, participants felt their asthma was fairly well controlled; $9 \%$ of Black participants, $11 \%$ of Hispanic participants, $7 \%$ of White participants, $10 \%$ of participants with low income, and $8 \%$ of participants with higher income indicated that their asthma was poorly or not at all controlled. More than $80 \%$ of participants in all subgroups reported that their asthma was currently under control (Fig. 3a). A higher percentage of Hispanic participants (41\%) than Black participants (36\%) and White participants $(30 \%)$, and a higher percentage of participants with low income $(42 \%)$ than those with higher income (31\%), reported that they used a rescue inhaler at least once a day in the past 4 weeks. Only $37 \%$ of all participants with asthma had an asthma action plan that was updated yearly. Hispanic participants were more likely than White participants to have an asthma action plan that was updated yearly (Fig. 3b). Participants with low income were less likely than participants with higher income to have an asthma action plan and to discuss asthma control with their HCP (Fig. 3b, c). A higher percentage of Black participants than White participants believe that their HCP always understands their needs for managing their asthma (Fig. 4). However, 14\% of Hispanic participants indicated that their HCP rarely or never understood their needs.

\section{Asthma Treatment}

Most (65\%) of the participants with asthma indicated that they see their primary care physician for asthma care. Black participants indicated they were more likely to receive care in an urgent care center for their asthma than White participants (9\% vs 3\%, respectively), whereas Hispanic participants indicated they were more likely to receive asthma care in the ED than White participants $(6 \%$ vs $2 \%$, respectively).

\section{Asthma Outcomes}

In the past 12 months, $39 \%$ of participants with asthma reported at least one visit to the ED/urgent care for asthma and 23\% had been hospitalized. Black and Hispanic participants were more likely than White participants to have at least one ED/urgent care visit or hospitalization for asthma in the past 12 months (Fig. 5). The percentage of participants with low income who had at least one ED/urgent care visit or hospitalization was similar to those with higher income (Fig. 5).

\section{Patient Perception of Eczema}

Approximately $20 \%$ of participants with eczema reported experiencing itchy and flaky skin and $36 \%$ experience dry/rough skin on a daily basis (Table 5). The daily occurrence of itchy, 
Table 2 Participant perception of the social impact of asthma or eczema

\begin{tabular}{llllllll}
\hline Social & Total with & Total with & White & Black & Hispanic & Low income High income \\
perception, & asthma & eczema & $(n=421)$ & $(n=252)$ & $(n=95)$ & $(n=99)$ & $(n=713)$ \\
$\%$ & $(n=554)$ & $(n=398)$ & & & & & \\
\hline
\end{tabular}

"Because of my asthma/eczema, I feel left out of things"

$\begin{array}{llllllll}\text { Never } & 38 & 61 & 56 & 42^{\mathrm{a}} & 39^{\mathrm{a}} & 40 & 48 \\ \text { Rarely } & 25 & 20 & 22 & 26 & 21 & 27 & 22 \\ \text { Sometimes } & 26 & 13 & 18 & 22 & 24 & 24 & 26 \\ \text { Often } & 8 & 5 & 3 & 8^{\mathrm{a}} & 12^{\mathrm{a}} & 8 & 6 \\ \text { Always } & 3 & 1 & 1 & 2 & 4 & 1 & 2\end{array}$

"Because of my asthma/eczema, people are unkind to me"

$\begin{array}{llllllll}\text { Never } & 68 & 78 & 75 & 68^{\mathrm{a}} & 66 & 64 & 73 \\ \text { Rarely } & 17 & 14 & 17 & 17 & 18 & 22 & 15 \\ \text { Sometimes } & 11 & 7 & 6 & 12^{\mathrm{a}} & 11^{\mathrm{a}} & 13 & 9 \\ \text { Often } & 2 & 0 & 1 & 1 & 3 & 0 & 2 \\ \text { Always } & 2 & 1 & 1 & 2 & 2 & 1 & 1\end{array}$

"Because of my asthma/eczema, people avoid looking at me"

$\begin{array}{llllllll}\text { Never } & 75 & 73 & 72 & 77 & 74 & 67 & 75 \\ \text { Rarely } & 12 & 19 & 20 & 9 & 11 & 20 & 14 \\ \text { Sometimes } & 10 & 6 & 7 & 10 & 13^{\mathrm{a}} & 10 & 8 \\ \text { Often } & 2 & 1 & 1 & 2 & 2 & 1 & 2 \\ \text { Always } & 1 & 1 & 0 & 2 & 0 & 2 & 1\end{array}$

"I feel embarrassed because of my asthma/eczema"

$\begin{array}{llllllll}\text { Never } & 52 & 35 & 40 & 59^{\mathrm{a}} & 48 & 50 & 46 \\ \text { Rarely } & 19 & 24 & 23 & 17^{\mathrm{a}} & 21 & 16 & 21 \\ \text { Sometimes } & 19 & 29 & 26 & 15^{\mathrm{a}} & 20 & 23 & 23 \\ \text { Often } & 7 & 8 & 7 & 7 & 5 & 5 & 7 \\ \text { Always } & 3 & 4 & 4 & 2 & 6 & 6 & 3\end{array}$

"I feel embarrassed because of my physical limitations

$\begin{array}{llllllll}\text { Never } & 44 & 47 & 46 & 53 & 46 & 37 & 48 \\ \text { Rarely } & 15 & 18 & 18 & 12^{\mathrm{a}} & 10^{\mathrm{a}} & 16 & 15 \\ \text { Sometimes } & 27 & 24 & 25 & 21 & 27 & 30 & 25 \\ \text { Often } & 7 & 7 & 6 & 6 & 7 & 8 & 7 \\ \text { Always } & 7 & 4 & 5 & 8 & 10^{\mathrm{a}} & 9 & 5\end{array}$

"Some people act as though it is my fault that I have asthma/eczema" 
Table 2 continued

\begin{tabular}{llllllll}
\hline $\begin{array}{l}\text { Social } \\
\text { perception, } \\
\text { \% }\end{array}$ & $\begin{array}{l}\text { Total with } \\
\text { asthma } \\
(\boldsymbol{n}=\mathbf{5 5 4})\end{array}$ & $\begin{array}{l}\text { Total with } \\
\text { eczema } \\
(\boldsymbol{n}=\mathbf{3 9 8})\end{array}$ & $\begin{array}{l}\text { White } \\
(\boldsymbol{n}=\mathbf{4 2 1})\end{array}$ & $\begin{array}{l}\text { Black } \\
(\boldsymbol{n}=\mathbf{2 5 2})\end{array}$ & $\begin{array}{l}\text { Hispanic } \\
(\boldsymbol{n}=\mathbf{9 5})\end{array}$ & $\begin{array}{l}\text { Low income } \\
(\boldsymbol{n}=\mathbf{9 9})\end{array}$ & $\begin{array}{l}\text { High income } \\
(\boldsymbol{n}=\mathbf{7 1 3})\end{array}$ \\
\hline Never & 62 & 69 & 68 & 66 & 62 & 62 & 66 \\
Rarely & 12 & 13 & 14 & 9 & 10 & 17 & 11 \\
Sometimes & 16 & 13 & 12 & 16 & 18 & 15 & 14 \\
Often & 7 & 4 & 5 & 7 & 7 & 4 & 6 \\
Always & 3 & 1 & 5 & 2 & 3 & 2 & 3 \\
\hline
\end{tabular}

${ }^{a}$ Significantly different than White participants

Table 3 Percentage of participants that stopped or modified their activities because of asthma

\begin{tabular}{lllllll}
\hline Activity, \% & $\begin{array}{l}\text { Total with asthma } \\
(\boldsymbol{n}=\mathbf{5 5 4})\end{array}$ & $\begin{array}{l}\text { White } \\
(\boldsymbol{n}=\mathbf{2 0 1})\end{array}$ & $\begin{array}{l}\text { Black } \\
(\boldsymbol{n}=\mathbf{2 1 3})\end{array}$ & $\begin{array}{l}\text { Hispanic } \\
(\boldsymbol{n}=\mathbf{8 3})\end{array}$ & $\begin{array}{l}\text { Low income } \\
(\boldsymbol{n}=\mathbf{7 4})\end{array}$ & $\begin{array}{l}\text { High income } \\
(\boldsymbol{n}=\mathbf{4 5 4})\end{array}$ \\
\hline Exercise & 42 & 42 & 43 & 51 & 41 & 44 \\
Sports & 25 & 21 & $31^{\mathrm{a}}$ & $34^{\mathrm{a}}$ & 28 & 25 \\
Climbing stairs & 19 & 24 & $16^{\mathrm{a}}$ & 19 & 23 & 19 \\
Parking closer to the door & 16 & 22 & $12^{\mathrm{a}}$ & $10^{\mathrm{a}}$ & 19 & 16 \\
$\quad$ when shopping & & & & & & \\
Social activities & 16 & 13 & 16 & 13 & 19 & 16 \\
Traveling & 14 & 17 & 14 & 13 & 18 & 14 \\
Shopping & 11 & 10 & 11 & 10 & 15 & 11 \\
Church & 5 & 5 & 5 & 4 & 4 & 5 \\
Movies & 4 & 3 & 3 & 4 & 5 & 4 \\
Other & 5 & 5 & 4 & $0^{\mathrm{a}}$ & 3 & 5 \\
None of these & 36 & 37 & 34 & 35 & 32 & 36 \\
\hline
\end{tabular}

${ }^{a}$ Significantly different than White participants

cracked, flaky, or dry/rough skin was reported in more Hispanic participants, but fewer Black participants, than White participants (Table 5). Participants with low income indicated that they experienced itchy, bleeding, weeping, and dry/rough skin more frequently than participants with higher income (Table 5). Among all participants with eczema, dry skin was the most common trigger that made eczema worse (76\%), followed by skin irritants (58\%) and stress (49\%). Nearly half of participants (45\%) reported sleep was disturbed at least 1-2 days a week because of their eczema. 
Table 4 Percentage of participants reporting "very much" or "a lot" for frequency of problems with eczema over the last week

\begin{tabular}{|c|c|c|c|c|c|c|}
\hline Problem, \% & $\begin{array}{l}\text { Total with } \\
\text { eczema } \\
(n=398)\end{array}$ & $\begin{array}{l}\text { White } \\
(n=298)\end{array}$ & $\begin{array}{l}\text { Black } \\
(n=54)\end{array}$ & $\begin{array}{l}\text { Hispanic } \\
(n=18)\end{array}$ & $\begin{array}{l}\text { Low } \\
\text { income } \\
(n=37)\end{array}$ & $\begin{array}{l}\text { High } \\
\text { income } \\
(n=350)\end{array}$ \\
\hline $\begin{array}{l}\text { How itchy, sore, painful or stinging has your skin } \\
\text { been? }\end{array}$ & 32 & 30 & 32 & 39 & 41 & 32 \\
\hline $\begin{array}{l}\text { How much has your skin influenced the clothes } \\
\text { you wear? }\end{array}$ & 16 & 14 & 17 & $50^{a}$ & 27 & 16 \\
\hline $\begin{array}{l}\text { How embarrassed or self-conscious have you } \\
\text { been because of your skin? }\end{array}$ & 14 & 12 & 15 & $33^{a}$ & 19 & 14 \\
\hline $\begin{array}{l}\text { How much has your skin interfered with you } \\
\text { going shopping or looking after your home or } \\
\text { garden? }\end{array}$ & 6 & 4 & 6 & $33^{\mathrm{a}}$ & 8 & 6 \\
\hline $\begin{array}{l}\text { How much has your skin affected any social or } \\
\text { leisure activities? }\end{array}$ & 6 & 5 & 6 & $17^{\mathrm{a}}$ & 8 & 6 \\
\hline $\begin{array}{l}\text { How much has your skin made it difficult for } \\
\text { you to do any sport? }\end{array}$ & 6 & 5 & 7 & $33^{\mathrm{a}}$ & 5 & 6 \\
\hline $\begin{array}{l}\text { How much of a problem has the treatment for } \\
\text { your skin been, for example by making your } \\
\text { home messy or by taking up time? }\end{array}$ & 6 & 5 & 6 & $17^{\mathrm{a}}$ & 5 & 6 \\
\hline $\begin{array}{l}\text { How much has your skin created problems with } \\
\text { your partner or close friends or relatives? }\end{array}$ & 4 & 3 & 4 & $22^{\mathrm{a}}$ & 0 & 4 \\
\hline $\begin{array}{l}\text { How much has your skin caused any sexual } \\
\text { difficulties? }\end{array}$ & 4 & 4 & 4 & $33^{a}$ & 5 & 4 \\
\hline
\end{tabular}

Questions are from the Dermatology Life Quality Index

${ }^{\text {a }}$ Significantly different than White participants

\section{Eczema Treatment}

Topical treatments, namely corticosteroids, were the most common form of treatment; $26 \%$ reported having tried systemic treatments (Supplemental Table E2). Use of calcineurin inhibitors and dupilumab was reported by a higher percentage of Hispanic participants than White participants (Supplemental Table E2). Participants with low income were more likely to have not tried any treatment for their eczema than participants with higher income (35\% vs $15 \%$, respectively).

\section{DISCUSSION}

The results of this survey indicate that patients with asthma and eczema perceive a physical, emotional, and social burden related to their condition. The burden of asthma and eczema disease and treatment impacts patients differently. These differences may be associated with various factors such as social determinates of health. Individuals who identified as Black or Hispanic endorsed barriers related to ineffective health education and communication strategies, such as a greater need for help filling out paperwork and problems understanding written 


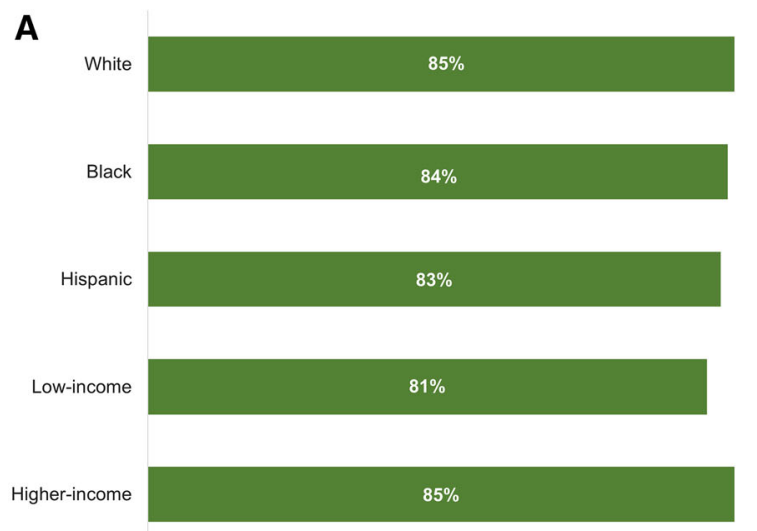

B

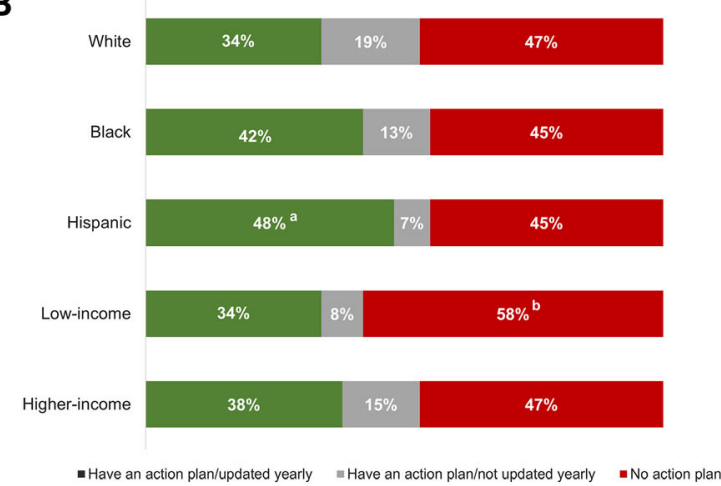

C

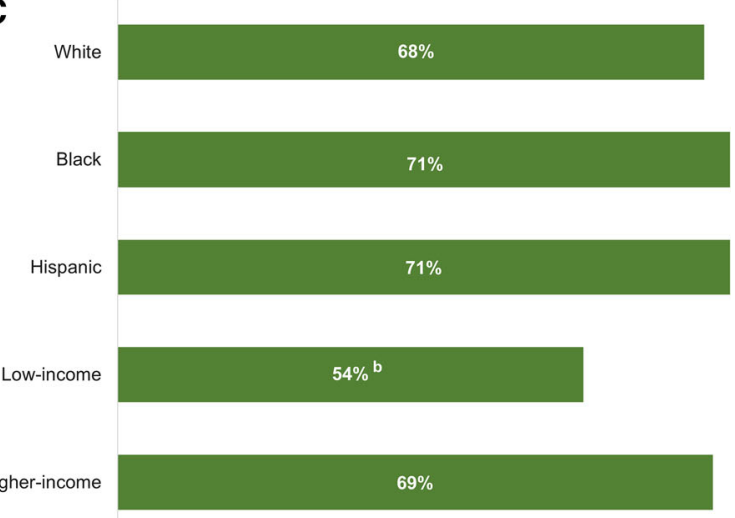

Fig. 3 Percentage of participants a who consider their asthma currently under control, $\mathbf{b}$ with an asthma action plan, and c who discuss asthma control with their physician. ${ }^{a}$ Significantly different than White participants. ${ }^{b}$ Significantly different than participants with higher income

materials, than White patients. Black and Hispanic participants also reported experiencing more ED visits and hospitalizations than White

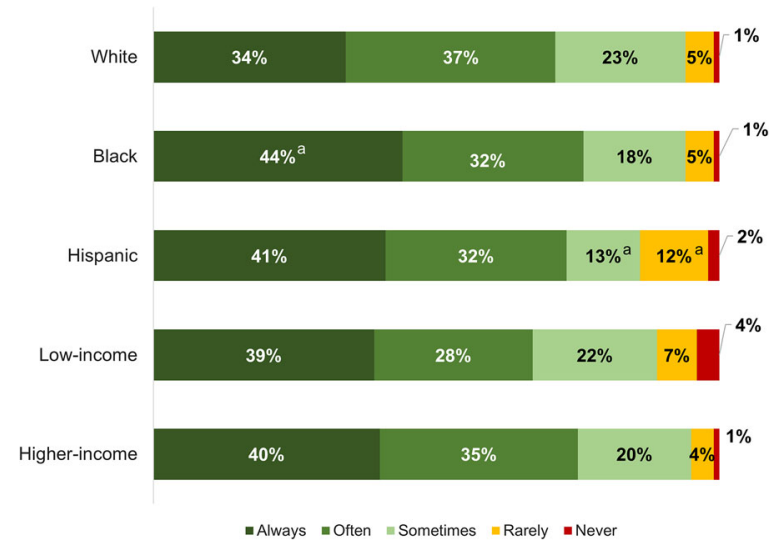

Fig. 4 Percentage of participants who believe their physician understands their needs for managing their asthma. ${ }^{a}$ Significantly different than White participants

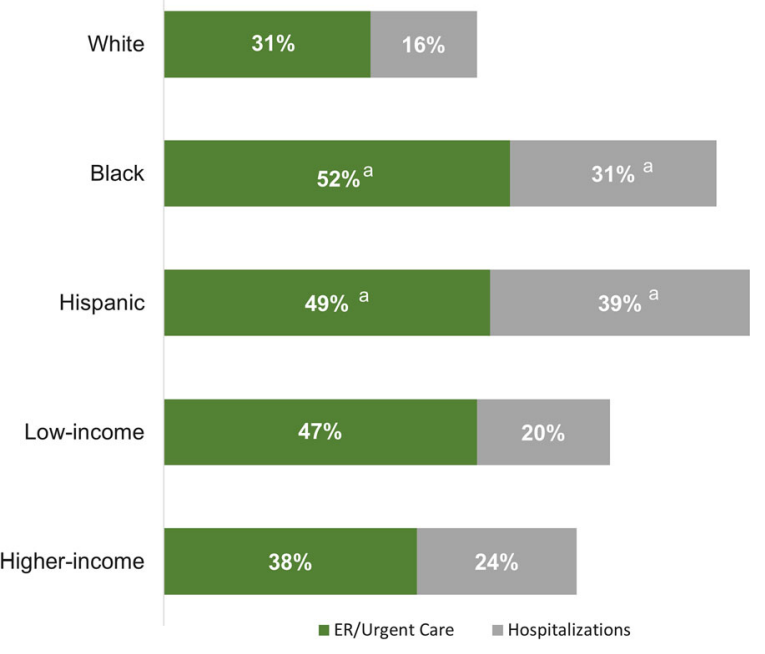

Fig. 5 Percentage of participants with at least one emergency department (ED)/urgent care visit or hospitalization for asthma in the past 12 months. ${ }^{\text {a Significantly }}$ different than White participants

participants. Patients reporting low income also endorse ineffective health education and communication strategies, have fewer asthma management discussions with an HCP, and have undermanaged eczema compared with patients with higher incomes. Thus, there is a need to develop effective and culturally appropriate teaching methods, materials, and interventions which meet the needs of all patients. Fortunately, nearly all of the participants had some form of health insurance, which was often 
Table 5 Percentage of participants reporting eczema symptoms

\begin{tabular}{lllllll}
\hline $\begin{array}{l}\text { Frequency of } \\
\text { symptoms, } \%\end{array}$ & $\begin{array}{l}\text { Total with eczema } \\
(n=398)\end{array}$ & $\begin{array}{l}\text { White } \\
(n=298)\end{array}$ & $\begin{array}{l}\text { Black } \\
(n=54)\end{array}$ & $\begin{array}{l}\text { Hispanic } \\
(n=18)\end{array}$ & $\begin{array}{l}\text { Low income } \\
(n=37)\end{array}$ & $\begin{array}{l}\text { High } \\
(n=3\end{array}$ \\
\hline $\begin{array}{l}\text { Has skin been itchy? } \\
\text { No days }\end{array}$ & 13 & 15 & 7 & 11 & 2 & 13 \\
$1-2$ days & 28 & 26 & 35 & 11 & 32 & 28 \\
$3-4$ days & 26 & 25 & 35 & 28 & 30 & 26 \\
$5-6$ days & 11 & 10 & 13 & $33^{\mathrm{a}}$ & 5 & 11 \\
Every day & 22 & 24 & $9^{\mathrm{a}}$ & 16 & 30 & 21
\end{tabular}

Has skin been flaking off?

$\begin{array}{ll}\text { No days } & 30 \\ 1-2 \text { days } & 28 \\ 3-4 \text { days } & 14 \\ \text { 5-6 days } & 7\end{array}$

Every day 21

Has skin felt dry/rough?

$\begin{array}{ll}\text { No days } & 12 \\ 1-2 \text { days } & 24 \\ 3-4 \text { days } & 17 \\ 5-6 \text { days } & 11 \\ \text { Every day } & 36\end{array}$

Has skin been cracked?

$\begin{array}{ll}\text { No days } & 39 \\ 1-2 \text { days } & 25 \\ 3-4 \text { days } & 14 \\ 5-6 \text { days } & 5 \\ \text { Every day } & 17\end{array}$

Has skin been bleeding?

$\begin{array}{ll}\text { No days } & 66 \\ 1-2 \text { days } & 22 \\ \text { 3-4 days } & 7 \\ 5-6 \text { days } & 3 \\ \text { Every day } & 2\end{array}$

Has skin been weeping?

No days $\quad 71$

$\begin{array}{lllll}30 & 43 & 17 & 24 & 30 \\ 29 & 28 & 11 & 35 & 28 \\ 12 & 17 & 33^{\mathrm{a}} & 14 & 14 \\ 7 & 3 & 11 & 8 & 7 \\ 22 & 9^{\mathrm{a}} & 28 & 19 & 21\end{array}$

High income

$(n=350)$ 
Table 5 continued

\begin{tabular}{lllllll}
\hline $\begin{array}{l}\text { Frequency of } \\
\text { symptoms, \% }\end{array}$ & $\begin{array}{l}\text { Total with eczema } \\
(\boldsymbol{n}=\mathbf{3 9 8})\end{array}$ & $\begin{array}{l}\text { White } \\
(\boldsymbol{n}=\mathbf{2 9 8})\end{array}$ & $\begin{array}{l}\text { Black } \\
(\boldsymbol{n}=\mathbf{5 4})\end{array}$ & $\begin{array}{l}\text { Hispanic } \\
(\boldsymbol{n}=\mathbf{1 8})\end{array}$ & $\begin{array}{l}\text { Low income } \\
(\boldsymbol{n}=\mathbf{3 7})\end{array}$ & $\begin{array}{l}\text { High income } \\
(\boldsymbol{n}=\mathbf{3 5 0})\end{array}$ \\
\hline 1-2 days & 18 & 20 & 11 & 22 & 26 & 18 \\
$3-4$ days & 7 & 6 & 7 & $22^{\mathrm{a}}$ & 11 & 7 \\
5-6 days & 2 & 1 & 2 & 6 & 3 & 2 \\
Every day & 2 & 2 & 0 & 0 & 0 & 1 \\
Has sleep been disturbed? & & & & & 57 \\
No days & 55 & 58 & 54 & $28^{\mathrm{a}}$ & 43 & 23 \\
1-2 days & 24 & 24 & 20 & 17 & 30 & 80 \\
3-4 days & 12 & 10 & $22^{\mathrm{a}}$ & $44^{\mathrm{a}}$ & 19 & 4 \\
$5-6$ days & 4 & 3 & 4 & 6 & 0 & 5 \\
Every day & 5 & 5 & 0 & 6 & 8 & \\
\hline
\end{tabular}

${ }^{a}$ Significantly different than White participants

bignificantly different than participants with higher income

provided by Medicaid or other state-funded programs.

The responses in this survey suggest that current methods of providing care to patients do not lead to equitable healthcare access to all. Participants with asthma reporting low income were also less likely to have an asthma action plan or discuss asthma control with their HCP, and $14 \%$ of Hispanic participants indicated that their HCP rarely or never understood their needs. Furthermore, among the participants with eczema, approximately a third of participants with low income and Hispanic participants had not tried any treatments for their eczema, despite a high frequency of symptoms and a negative impact of their eczema on quality of life. These results emphasize the importance of effective communication, including addressing language barriers and implementation of shared decision-making (SDM) between HCPs and patients in asthma and eczema disease management. Although SDM should be a part of disease management for every patient, more research is needed to understand the needs of patients with low income and how SDM may meet these needs. Shared decision-making takes patient preferences and limitations into account, giving patients a sense of empowerment for their treatment and engaging the HCP to discover what the patient actually needs, not what the HCP thinks the patient needs. Surveys of a diverse patient population in Canada revealed that total family income was the only nonmodifiable patient characteristic that was associated with an SDM experience, with higher income being associated with a better experience [18]. Patients with low income or those from marginalized groups may feel less empowered to facilitate SDM or be less likely to be seen in environments where SDM is focused on as a priority in interacting with patients. Thus, greater efforts are needed to implement SDM in clinics where it is not currently being practiced and to make SDM easier for patients. SDM will further inform caregivers on the individualized burden of disease and burden of therapy.

Among the participants of this survey, Black and Hispanic participants were more likely to 
have an ED visit or hospitalization for asthma within the last 12 months than White participants. ED visits are an indicator of poor asthma control because they are often associated with an exacerbation. However, participants generally perceived their asthma as being fairly well controlled and the survey did not capture the reasons that individuals were seen in the ED versus another type of medical setting. A crosssectional study of older patients with asthma in the USA also found a higher number of ED visits in Black and Hispanic individuals compared with White individuals even after controlling for medication use, health insurance coverage, and impaired access to cost [1]. It is possible that some patients may be limited to seeking care in the ED because of a lack of HCPs in predominantly Black, Hispanic, and low-income communities or because of a limited number of medical care sites that accept their insurance. Social determinants of health overall may drive higher hospitalizations in marginalized minority populations. A study of COVID19 cases in a California integrated health system found that Black patients had a 2.7-times higher likelihood of hospitalization than non-Hispanic White patients after controlling for age, sex, comorbidities, and income [19]. Furthermore, social and economic vulnerability is significantly associated with COVID-19 incidence and mortality $[20,21]$. Racism and provider bias in the healthcare system are causes for healthcare disparities that are beginning to be explored for asthma and eczema [22-25]. Although genetic factors have often been hypothesized as a reason for the disparity in health outcomes in Black patients, an analysis of two large cohorts in the USA indicated that African genetic ancestry was not predictive of risk of eczema or eczema control [26].

The results of the current survey indicate that disparities in asthma and eczema patients occur on physical, economic, and emotional levels. Overall health is influenced not just by the disease itself but societal factors including housing, environmental quality, food security, and discrimination/racism. To achieve health equity in asthma and eczema, these societal factors need to be addressed (Fig. 6) [27].

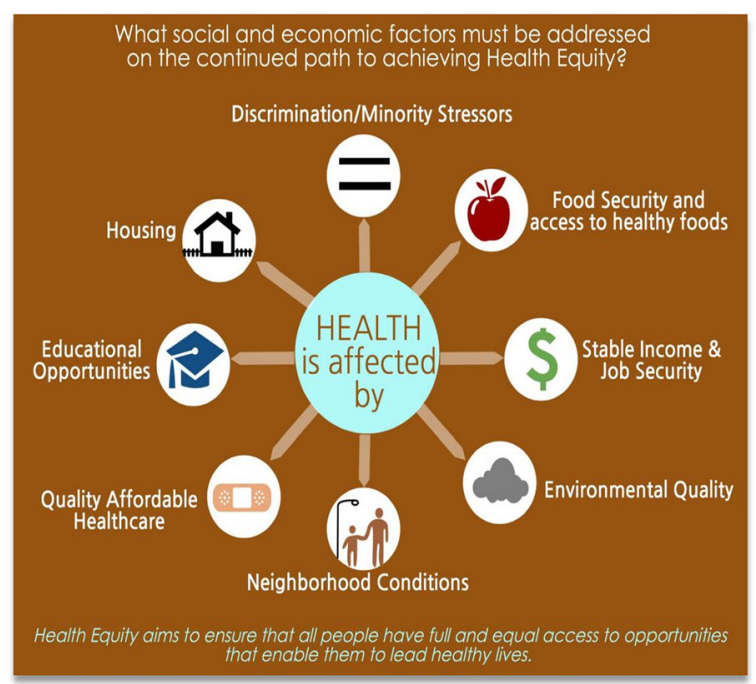

Fig. 6 The path to achieving health equity. Adapted and reproduced from the Health Equity Institute for Research, Practice \& Policy [27]

A limitation of the survey is that it only examined White, Black, or Hispanic, and lowincome versus higher-income subpopulations. No analyses within Hispanic subgroups (e.g., Black Hispanic) were conducted, and other subpopulations in the USA that have been shown to be at-risk for poor asthma and eczema outcomes (i.e., American Indian/Alaska Native, maternal smoking) were not evaluated [28, 29]. The numbers of Hispanic and participants with low income were relatively low compared with the other subpopulations, which may limit the ability to make effective comparisons. In addition, since participants were reached by online panels and internet access was required to complete the survey, participant selection was limited and potentially biased to those with access to these resources.

\section{CONCLUSIONS}

Healthcare programs, payers, and policy makers need to be aware of the populations at-risk for the greatest burden associated with asthma and eczema. Specific efforts, including more effective and culturally informed communication and education strategies to improve health information uptake and SDM, need to be made 
to provide the resources and support to reduce the burdens of disease and treatment in these highly impacted populations.

\section{ACKNOWLEDGEMENTS}

We would like to thank the participants for their time and effort to take the survey.

Funding. The survey was funded by the Allergy \& Asthma Network, Vienna, VA, USA and supported by Sanofi/Regeneron. The journal Rapid Service and Open Access fees were funded by Allergy \& Asthma Network.

Medical Writing, Editorial, and Other Assistance. Medical writing and editorial assistance were provided by Erin P. Scott, PhD, of Scott Medical Communications, LLC. This assistance was funded by the Allergy \& Asthma Network.

Authorship. All named authors meet the International Committee of Medical Journal Editors (ICMJE) criteria for authorship for this article, take responsibility for the integrity of the work as a whole, and have given their approval for this version to be published.

Author Contributions. Don A. Bukstein contributed to the interpretation the data and reviewing and editing of the manuscript. Adam Friedman contributed to the interpretation the data and reviewing and editing of the manuscript. Erika Gonzalez Reyes contributed to the interpretation the data and reviewing and editing of the manuscript. Bridgette Jones contributed to the interpretation the data and reviewing and editing of the manuscript. Mary Hart contributed to the development of the survey, to the interpretation of the results, and reviewing and editing of the manuscript. Tonya Winders contributed to the development of the survey, to the interpretation of the results, and reviewing and editing of the manuscript.

Disclosures. Don A. Bukstein has served as a speaker for ALK, AstraZeneca, Circassia, Genentech, Novartis, and Teva, has received research support from Genentech and Novartis, has served as an investigator for Allergy, Asthma \& Sinus Center, and has other commercial interests in The PBL Institute, LLC and AltusLearns, LLC. Adam Friedman is a speaker and consultant for Regeneron and Sanofi Genzyme and has served on advisory boards for Aveeno, LRP, and Pfizer. Erika Gonzalez Reyes has served on an advisory board for GlaxoSmithKline and has served as an investigator for TEVA, ALK, Regeneron, Genentech, and Novartis. Mary Hart has nothing to disclose. Bridgette Jones serves as a paid author for Merck Manuals. Tonya Winders has nothing to disclose; however, her employer Allergy \& Asthma Network has received funding from Sanofi/Regeneron for unbranded disease awareness and education.

Compliance with Ethics Guidelines. The survey was reviewed by an institutional review board and was granted exemption status. All participants provided written informed consent at the time of survey participation for the use of their survey responses.

Data Availability. The datasets generated during and/or analyzed during the current study are available from the corresponding author on reasonable request.

Open Access. This article is licensed under a Creative Commons Attribution-NonCommercial 4.0 International License, which permits any non-commercial use, sharing, adaptation, distribution and reproduction in any medium or format, as long as you give appropriate credit to the original author(s) and the source, provide a link to the Creative Commons licence, and indicate if changes were made. The images or other third party material in this article are included in the article's Creative Commons licence, unless indicated otherwise in a credit line to the material. If material is not included in the article's Creative Commons licence and your intended use is not permitted by statutory regulation or exceeds the permitted use, you will need to obtain permission directly from the copyright holder. To view a copy of this licence, 
visit http://creativecommons.org/licenses/bync/4.0/.

\section{REFERENCES}

1. Cremer NM, Baptist AP. Race and asthma outcomes in older adults: results from the National Asthma Survey. J Allergy Clin Immunol Pract. 2020;8:1294301.e7.

2. Largent J, Nickerson B, Cooper D, Delfino RJ. Paediatric asthma hospital utilization varies by demographic factors and area socio-economic status. Public Health. 2012;126:928-36.

3. Mosen DM, Schatz M, Gold R, Mularski RA, Wong WF, Bellows J. Medication use, emergency hospital care utilization, and quality-of-life outcome disparities by race/ethnicity among adults with asthma. Am J Manag Care. 2010;16:821-8.

4. Silverberg JI, Gelfand JM, Margolis DJ, et al. Atopic dermatitis in US adults: from population to health care utilization. J Allergy Clin Immunol Pract. 2019;7:1524-32.e2.

5. Brunner PM, Guttman-Yassky E. Racial differences in atopic dermatitis. Ann Allergy Asthma Immunol. 2019;122:449-55.

6. Jothishankar B, Stein SL. Impact of skin color and ethnicity. Clin Dermatol. 2019;37:418-29.

7. Sullivan K, Thakur N. Structural and social determinants of health in asthma in developed economies: a scoping review of literature published between 2014 and 2019. Curr Allergy Asthma Rep. 2020;20:5

8. Sitarik A, Havstad S, Kim H, et al. Racial disparities in allergic outcomes persist to age 10 years in black and white children. Ann Allergy Asthma Immunol. 2020;124:342-9.

9. Whiteley J, Emir B, Seitzman R, Makinson G. The burden of atopic dermatitis in US adults: results from the 2013 National Health and Wellness Survey. Curr Med Res Opin. 2016;32(10):1645-51.

10. Wei W, Ghorayeb E, Andria M, et al. A real-world study evaluating adeQUacy of Existing Systemic Treatments for patients with moderate-to-severe Atopic Dermatitis (QUEST-AD): baseline treatment patterns and unmet needs assessment. Ann Allergy Asthma Immunol. 2019;123:381-8.e2.

11. Meltzer EO, Blaiss MS, Nathan RA, Doherty DE, Murphy KR, Stoloff SW. Asthma burden in the
United States: results of the 2009 Asthma Insight and Management survey. Allergy Asthma Proc. 2012;33:36-46.

12. Sanz de Burgoa V, Rejas J, Ojeda P. Self-perceived Sleep quality and quantity in adults with asthma: findings from the CosteAsma Study. J Investig Allergol Clin Immunol. 2016;2(6):256-62.

13. Drucker AM, Qureshi AA, Amand C, et al. Health care resource utilization and costs among adults with atopic dermatitis in the United States: a claims-based analysis. J Allergy Clin Immunol Pract. 2018;6:1342-8.

14. Teasdale E, Muller I, Sivyer K, et al. Views and experiences of managing eczema: systematic review and thematic synthesis of qualitative studies. $\mathrm{Br} \mathrm{J}$ Dermatol. 2021;184(4):627-37.

15. Peláez S, Lamontagne AJ, Collin J, et al. Patients' perspective of barriers and facilitators to taking long-term controller medication for asthma: a novel taxonomy. BMC Pulm Med. 2015;15:42.

16. Nurmagambetov T, Kuwahara R, Garbe P. The economic burden of asthma in the United States, 2008-2013. Ann Am Thorac Soc. 2018;15:348-56.

17. Sullivan PW, Slejko JF, Ghushchyan VH, et al. The relationship between asthma, asthma control and economic outcomes in the United States. J Asthma. 2014;51:769-78.

18. Manhas KP, Olson K, Churchill K, Faris P, Vohra S, Wasylak T. Measuring shared decision-making and collaborative goal setting in community rehabilitation: a focused ethnography using cross-sectional surveys in Canada. BMJ Open. 2020;10:e034745.

19. Azar MJ, Shen Z, Romanelli RJ, et al. Disparities in outcomes among COVID-19 patients in a large health care system in California. Health Aff. 2020;39:1253-62.

20. Sandhu A, Korzeniewski SJ, Polistico J, et al. Elevated COVID19 mortality risk in Detroit area hospitals among patients from census tracts with extreme socioeconomic vulnerability. EClinicalMedicine. 2021;34:100814.

21. Khazanchi R, Beiter ER, Gondi S, Beckman AL, Bilinski A, Ganguli I. County-level association of social vulnerability with COVID-19 cases and deaths in the USA. J Gen Intern Med. 2020;35: 2784-7.

22. Louisias M, Matsui E. Disentangling the root causes of racial disparities in asthma: the role of structural racism in a 5-year-old black boy with uncontrolled asthma. J Allergy Clin Immunol Pract. 2020;8: 1162-4. 
23. Sabin JA, Greenwald AG. The influence of implicit bias on treatment recommendations for 4 common pediatric conditions: pain, urinary tract infection, attention deficit hyperactivity disorder, and asthma. Am J Public Health. 2012;102:988-95.

24. Carlson S, Borrell LN, Eng C, et al. Self-reported racial/ethnic discrimination and bronchodilator response in African American youth with asthma. PLOS ONE. 2017;12:e0179091.

25. Tackett KJ, Jenkins F, Morrell DS, McShane DB, Burkhart CN. Structural racism and its influence on the severity of atopic dermatitis in African American children. Pediatr Dermatol. 2020;37:142-6.

26. Abuabara K, You Y, Margolis DJ, Hoffmann TJ, Risch N, Jorgenson E. Genetic ancestry does not explain increased atopic dermatitis susceptibility or worse disease control among African American subjects in 2 large US cohorts. J Allergy Clin Immunol. 2020;145:192-8.e11.

27. Health Equity Institute. The path to achieving health equity. https://dev-sfsu-healthequity. pantheonsite.io/sites/default/files/images/

infographic-path-to-health-equity.jpg. Accessed 30 July 2021 .

28. Wen C, Liu SH, Li Y, Sheffield P, Liu B. Pediatric asthma among small racial/ethnic minority groups: an analysis of the 2006-2015 National Health Interview Survey. Public Health Rep. 2019;134: 338-43.

29. Rutter CE, Silverwood RJ, Asher MI, et al. Comparison of individual-level and population-level risk factors for rhinoconjunctivitis, asthma, and eczema in the International Study of Asthma and Allergies in Childhood (ISAAC) Phase Three. World Allergy Organ J. 2020;13:100123. 